\title{
BMJ Open Neuropsychiatric and cardiometabolic comorbidities in patients with previously diagnosed Cushing's disease: a longitudinal observational study
}

\author{
C Dimopoulou, V Geraedts, G K Stalla, C Sievers
}

To cite: Dimopoulou C, Geraedts V, Stalla GK, et al. Neuropsychiatric and cardiometabolic comorbidities in patients with previously diagnosed Cushing's disease: a longitudinal observational study. BMJ Open 2015;5: e006134. doi:10.1136/ bmjopen-2014-006134

- Prepublication history for this paper is available online. To view these files please visit the journal online (http://dx.doi.org/10.1136/ bmjopen-2014-006134).

Received 17 July 2014 Revised 12 December 2014 Accepted 22 December 2014

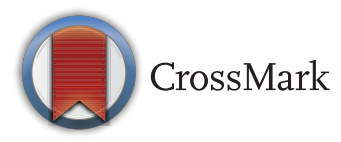

Department of Neuroendocrinology, Max Planck Institute of Psychiatry (MPIP), Munich, Germany

Correspondence to V Geraedts;

v.j.geraedts@umail. leidenuniv.nt

\section{ABSTRACT}

Introduction: Only few studies have systematically investigated neuropsychiatric aspects in patients with Cushing's disease (CD). Pain syndromes have been described in patients with pituitary adenomas, but so far no systematical investigation has been conducted in patients with $C D$. Additionally, $C D$ has an association with cardiometabolic comorbidities which ultimately leads to increased morbidity and mortality. Long-term treatment of the hypercortisolic state cannot prevent the persistence of an unfavourable cardiometabolic risk profile. Finally, chronic hypercortisolism is known to impact the health-related quality of life (HRQoL). We aim to systematically investigate the neuropsychiatric and cardiometabolic comorbidities, as well as assess the HRQoL, in patients with previously diagnosed $C D$ in a longitudinal fashion.

Methods and analysis: In this longitudinal study, we will assess 20 patients with $C D$ displaying biochemical control 24 months after recruitment in the initial cross-sectional study $(n=80)$. This will be a mixed cohort including patients after surgical, after radiation therapy and/or under current medical treatment for $\mathrm{CD}$. Primary outcomes include changes in mean urinary free cortisol and changes in specific pain patterns. Secondary/exploratory neuropsychiatric domains include depression, anxiety, personality, sleep, body image and quality of life. Secondary/ exploratory cardiometabolic domains include anthropometric parameters, cardiometabolic risk biomarkers and insulin resistance. Additional domains will be investigated if warranted by clinical indication. Safety assessment under medical therapy will include liver enzymes, ECG abnormalities and hyperglycaemia. Ethics and dissemination: Risk of damage from study-conditioned measures is very small and considered ethically justified. Dual-energy X-ray absorptiometry may call for detailed fracture risk assessment. However, the radiation dose is very small and only administered on clinical indication; therefore, it is considered ethically justified. This protocol has been approved by the local medical ethics committee.

\section{INTRODUCTION}

Clinical presentation of Cushing's disease (CD) reflects chronic cortisol excess and comprises a broad spectrum of features, among others psychiatric disorders such as depression and psychosis. ${ }^{1}$ Despite these clinical observations, only few studies have investigated systematically and with standardised instruments neuropsychiatric aspects in patients with $\mathrm{CD}$, such as psychopathology, ${ }^{2}$ neuropsychology, ${ }^{3}$ personality, sleep, pain, brain architecture ${ }^{4}$ and quality of life, ${ }^{5} 6$ and how these comorbidities and symptoms are affected by different therapy regimens. Moreover, it is known that patients with pituitary adenomas frequently suffer from pain syndromes, for example, headache $;^{7}$ however, this has not been systematically investigated in a subset of patients with CD to date. This is the main reason for selecting the change in pain patterns as a primary end point in this study. Additionally, recent studies suggest that chronic hypercortisolism promotes brain changes such as cortical frontal thinning and hippocampal dysfunction. ${ }^{8} 9$

Furthermore, $\mathrm{CD}$ is associated with increased morbidity and mortality, mostly due to cardiometabolic comorbidities such as arterial hypertension, glucose intolerance, diabetes mellitus, dyslipidaemia, thromboembolic complications as well as a hypercoagulable state. ${ }^{10-12}$ Treatment of hypercortisolism has been demonstrated to be associated with a significant reduction in mortality and morbidity. However, even after long-term cure of the disease (mean time of hormonal cure 11 \pm 6 years), patients exhibit a persistent accumulation of central fat comparable to active hypercortisolism, leading to a persistent and unfavourable cardiometabolic risk profile. ${ }^{13}$

Chronic exposure to hypercortisolism has a significant impact on patient's health and health-related quality of life (HRQoL), as demonstrated with generic questionnaires. Two disease-generated questionnaires, the CushingQoL and the Tuebingen CD-25, have been developed in order to evaluate HRQoL in patients with $\mathrm{CD}^{6}{ }^{14}{ }^{15}$ Interestingly, even 
after long-term remission of $\mathrm{CD}$ (duration of remission $13.3 \pm 10.4$ years), impaired quality of life persists as a remaining effect of long-standing hypercortisolism. ${ }^{16}$

The aim of this study is to systematically assess neuropsychiatric and cardiometabolic comorbidities, as well as HRQoL, in patients with CD displaying biochemical control 24 months after recruitment in the initial crosssectional study in order to scientifically verify clinical observations. This addresses a hiatus in our current understanding of CD and may ultimately lead to better patient management.

\section{METHOD}

\section{Study population}

Only few clinical data exist regarding the development/ improvement of neuropsychiatric comorbidities in patients with CD under treatment. Therefore, in order to determine the appropriate sample size, we cannot make use of any well-known or estimated variances or mean value differences of the target variables.

To determine the sample sizes that are needed to detect a difference between two proportions, an a priori power calculation has been conducted. Here, the expected proportion of mental disorders/pain syndromes in the study sample has been based on the aforementioned references. Specifying type I error as 0.05 and a power $\geq 80 \%, \quad(\delta=42, \sigma=28)$, a sample size of \pm 14 patients will be needed to detect the specified differences. Assuming a safe margin, we deem 20 patients to be more than sufficient for our study target.

Following previous rationale, we will recruit 20 (of the initial 80 patients enrolled in the cross-sectional part of the study) patients with previously diagnosed $\mathrm{CD}$, who will show biochemical control of the disease 24 months after study enrolment, including patients after surgical, after irradiation therapy and/or under current medical treatment for CD. Patients will be recruited from the endocrine outpatient clinic of the Max Planck Institute of Psychiatry, Munich and the Department of Internal Medicine, Ludwig-Maximilians-University, Munich.

Inclusion criteria include:

1. Adult patients with confirmed CD, who will show biochemical control of the disease 24 months after initial study enrolment including patients after surgical, before/under/after irradiation therapy and/or under current medical treatment.

2. Written informed consent.

Exclusion criteria include:

1. Female pregnant patients.

\section{Primary objectives}

In order to document arithmetic and percentage change in mean urinary free cortisol (UFC) values between uncontrolled state/before treatment (baseline) versus biochemical control of CD (2-year follow-up), we will measure the mean of absolute changes in UFC values in both groups.
In order to document arithmetic and percentage change in specific pain patterns between uncontrolled state/before treatment (baseline) versus biochemical control of CD (2-year follow-up), we will measure the change in painDETECT scores.

\section{Secondary/exploratory}

In order to document the change in neuropsychiatric comorbidities between uncontrolled/before treatment (baseline) versus biochemical control of CD (2-year follow-up), we assess the following domains with the associated standardised questionnaires:

- Depression using Beck's Depression Inventory (BDI);

- Anxiety using State-Trait Anxiety Inventory (STAI);

- Personality using Cloninger Temperament and Personality Questionnaire (TPQ) and Eysenck Personality Questionnaire (EPQ-RK);

- Daytime sleepiness using Epworth Sleepiness Scale (ESS);

- Subjective sleep using Pittsburgh Sleep Quality Index (PSQI);

- Specific pain patterns using Migraine Disability Assessment (MIDAS) and German Society for the Study of Pain (DGSS);

- Body image using Fragebogen zur Beurteilung des eigenen Körpers (FBeK) and Fragebogen zum Körperbild (FKB-20);

- HRQoL using SF-36, EuroQoL and CushingQoL;

- Sleep EEG, cognition and functional connectivity (MRI) (only in patients with a clinical indication, for example, severe cognitive impairment or severe sleep disorder as set in the former cross-sectional part of the study).

In order to document the change in cardiometabolic comorbidities between uncontrolled/before treatment (baseline) versus biochemical control of CD (2-year follow-up), we will assess the following domains:

- Anthropometric parameters including height, weight, body mass index, waist-and-hip circumference and waist-to-height ratio;

- Cardiometabolic risk biomarkers including fasting plasma glucose, glycosylated haemoglobin (HbAlc), triglycerides, total cholesterol, high-density lipoprotein, low-density lipoprotein and lipoprotein (a);

- Insulin resistance using the homoeostatic model assessment index;

- Bone mineral density, fat-free mass, fat mass and fat mass percentage using dual-energy X-ray absorptiometry (DXA) In addition, fracture risk assessment within the study population will be carried out with the FRAX algorithm (http://www.shef.ac.uk/FRAX) as suggested by Trementino et al; ${ }^{17}$

\section{- Safety.}

To assess the safety of current medical treatment for $\mathrm{CD}$, we will report all adverse and serious adverse events under treatment with severity graded according to the Common Toxicity Criteria for Adverse Events (CTCAE) 
V.3.0. If CTCAE grading does not exist, CTC grades 1-4 will be used (higher scores indicating greater severity).

Assessment of safety under current medical treatment will include:

- Assessment of liver enzymes (alanine transaminase (ALT), aspartate transaminase (AST), alkaline phosphatase (ALP) and total bilirubin);

- Assessment of ECG abnormalities (QTcF interval);

- Assessment of hyperglycaemia (fasting plasma, glucose, insulin, HbA1c).

Current medical treatment will be discontinued if:

- ALT or AST $>3 \times$ upper limit of the normal range (ULN) and total bilirubin $\geq 2 \times \mathrm{ULN}$ and ALP $<2 \times \mathrm{ULN}$;

- ALT or AST $>5 \times \mathrm{ULN}$ and $\leq 8 \times \mathrm{ULN}$ persistent for more than 2 weeks;

- ALT or AST $8 \times U L N$;

- QTcF >480 ms;

- Uncontrolled diabetes mellitus.

\section{ETHICS AND DISSEMINATION}

This is a longitudinal observational study. All patients will provide their written informed consent which can be withdrawn at any time during study participation.

The risk of study participants suffering damage from study-conditioned measures is very small and, from the sponsor's point of view, ethically justified. At the moment, there are no available guidelines for the management of osteoporosis induced by endogenous hypercortisolism. Within this study, bone mineral density will be measured by DXA and fracture risk will be assessed by the FRAX algorithm, according to recently published expert opinions and suggestions. ${ }^{18-20}$ The amount of radiation used in DXA is extremely small and therefore, according to our opinion, ethically justified.

This protocol has been approved by the local medical ethics committee.

\section{CONCLUSIONS}

Our research group has assessed 80 patients with $\mathrm{CD}$ in a cross-sectional setting. Results indicate that, in a crosssectional observational study, patients with $\mathrm{CD}$ were shown to have a particularly high susceptibility to pain ${ }^{21}$ and increased anxiety-associated personality traits. ${ }^{22}$ This study will expand on these previous findings.

Contributors CD and VG wrote the clinical study protocol. GS and CS were responsible for the design of the study, the inclusion of patients and the preparation of the study protocol.

Funding This work was supported by Novartis, NCC-Code: CSOM230BDE05T. Competing interests None.

Patient consent Obtained.

Ethics approval Ethical Committee Ludwig-Maximilians University.

Provenance and peer review Not commissioned; externally peer reviewed.
Open Access This is an Open Access article distributed in accordance with the Creative Commons Attribution Non Commercial (CC BY-NC 4.0) license, which permits others to distribute, remix, adapt, build upon this work noncommercially, and license their derivative works on different terms, provided the original work is properly cited and the use is non-commercial. See: http:// creativecommons.org/licenses/by-nc/4.0/

\section{REFERENCES}

1. Boscaro M, Barzon L, Fallo F, et al. Cushing's syndrome. Lancet 2001;357:783-91.

2. Dorn LD, Burgess ES, Friedman TC, et al. The longitudinal course of psychopathology in Cushing's syndrome after correction of hypercortisolism. J Clin Endocrinol Metab 1997;82:912-19.

3. Forget $\mathrm{H}$, Lacroix $\mathrm{A}$, Cohen $\mathrm{H}$. Persistent cognitive impairment following surgical treatment of Cushing's syndrome. Psychoneuroendocrinology 2002;27:367-83.

4. Bourdeau I, Bard C, Noel B, et al. Loss of brain volume in endogenous Cushing's syndrome and its reversibility after correction of hypercortisolism. J Clin Endocrinol Metab 2002;87:1949-54.

5. van Aken MO, Pereira AM, Biermasz NR, et al. Quality of life in patients after long-term biochemical cure of Cushing's disease. J Clin Endocrinol Metab 2005;90:3279-86.

6. Webb SM, Badia X, Barahona MJ, et al. Evaluation of health-related quality of life in patients with Cushing's syndrome with a new questionnaire. Eur J Endocrinol 2008;158:623-30.

7. Levy MJ, Matharu MS, Meeran K, et al. The clinical characteristics of headache in patients with pituitary tumours. Brain 2005;128 (Pt 8):1921-30.

8. Crespo I, Esther GM, Santos A, et al. Impaired decision-making and selective cortical frontal thinning in Cushing's syndrome. Clin Endocrinol (Oxf) 2014;81:826-33.

9. Resmini E, Santos A, Gómez-Anson B, et al. Hippocampal dysfunction in cured Cushing's syndrome patients, detected by (1) H-MR-spectroscopy. Clin Endocrinol (Oxf) 2013;79:700-7.

10. Boscaro M, Sonino N, Scarda A, et al. Anticoagulant prophylaxis markedly reduces thromboembolic complications in Cushing's syndrome. J Clin Endocrinol Metab 2002;87:3662-6.

11. Mancini T, Kola B, Mantero F, et al. High cardiovascular risk in patients with Cushing's syndrome according to $1999 \mathrm{WHO} / \mathrm{ISH}$ guidelines. Clin Endocrinol (Oxf) 2004;61:768-77.

12. Pivonello R, Faggiano A, Lombardi G, et al. The metabolic syndrome and cardiovascular risk in Cushing's syndrome. Endocrinol Metab Clin North Am 2005;34:327-39, viii.

13. Barahona MJ, Sucunza N, Resmini E, et al. Persistent body fat mass and inflammatory marker increases after long-term cure of Cushing's syndrome. J Clin Endocrinol Metab 2009;94:3365-71.

14. Milian M, Honegger J, Teufel $P$, et al. Tuebingen CD-25 is a sensitive tool to investigate health-related quality of life in Cushing's disease patients in the course of the disease. Neuroendocrinology 2013;98:188-99.

15. Nelson LM, Forsythe A, McLeod L, et al. Psychometric evaluation of the Cushing's Quality-of-Life questionnaire. Patient 2013;6:113-24.

16. Wagenmakers MA, Netea-Maier RT, Prins JB, et al. Impaired quality of life in patients in long-term remission of Cushing's syndrome of both adrenal and pituitary origin: a remaining effect of long-standing hypercortisolism? Eur J Endocrinol 2012;167:687-95.

17. Trementino L, Ceccoli L, Concettoni $C$, et al. Fracture risk assessment before and after resolution of endogenous hypercortisolism: is the $\operatorname{FAX}(\circledR)$ algorithm useful? $J$ Endocrinol Invest 2014;37:957-65.

18. Scillitani A, Mazziotti G, Di Somma C, et al. ABC Group. Treatment of skeletal impairment in patients with endogenous hypercortisolism: when and how? Osteoporos Int 2014;25:441-6.

19. Arnaldi G, Mancini T, Tirabassi G, et al. Advances in the epidemiology, pathogenesis, and management of Cushing's syndrome complications. J Endocrinol Invest 2012;35:434-48.

20. Tóth M, Grossman A. Glucocorticoid-induced osteoporosis: lessons from Cushing's syndrome. Clin Endocrinol (Oxf) 2013;79:1-11.

21. Dimopoulou C, Athanasoulia AP, Hanisch E, et al. Clinical characteristics of pain in patients with pituitary adenomas. Eur $\mathrm{J}$ Endocrinol 2014;171:581-91.

22. Dimopoulou C, Ising M, Pfister $\mathrm{H}$, et al. Increased prevalence of anxiety-associated personality traits in patients with Cushing's disease: a cross-sectional study. Neuroendocrinology 2013;97:139-45. 